Trouille, Jean-Marc and Trouille, Helen

ORCID: https://orcid.org/0000-0002-0383-8920 (2021) La question du Brexit sous la présidence allemande. D'une négociation à hauts risques vers un Brexit dur/The issue of Brexit during the German presidency - From a high-risk negotiation towards a hard Brexit. Allemagne d aujourd hui (236). pp. 91-102.

Downloaded from: http://ray.yorksj.ac.uk/id/eprint/5515/

The version presented here may differ from the published version or version of record. If you intend to cite from the work you are advised to consult the publisher's version: https://www.cairn.info/revue-allemagne-d-aujourd-hui-2021-2.htm

Research at York St John (RaY) is an institutional repository. It supports the principles of open access by making the research outputs of the University available in digital form. Copyright of the items stored in RaY reside with the authors and/or other copyright owners. Users may access full text items free of charge, and may download a copy for private study or non-commercial research. For further reuse terms, see licence terms governing individual outputs. Institutional Repository Policy Statement

\title{
RaY
}

Research at the University of York St John

For more information please contact RaY at ray@yorks.ac.uk 


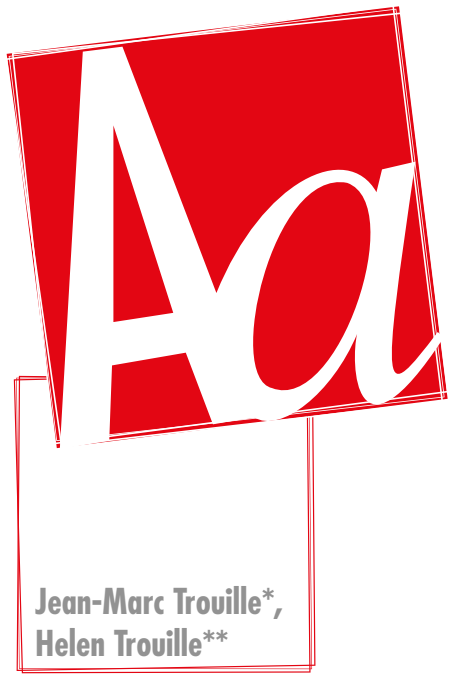

\section{La question du Brexit sous la présidence allemande. D'une négociation à hauts risques vers un Brexit dur}

Les six mois de la présidence allemande de l'Union Européenne (UE) ont véritablement constitué une période de détricotage entre I'UE et le Royaume-Uni. Ce dernier avait certes officiellement quitté I'Union le 31 janvier 2020, mais il n'en restait pas moins dans une phase de transition de onze mois, qui lui permettait de bénéficier de la protection du Marché intérieur et de l'Union douanière jusqu'au 31 décembre 2020 jusqu'à la conclusion des négociations commerciales avec I'UE. En 2017, Pascal Lamy, ancien directeur de l'Organisation Mondiale du Commerce, avait comparé ce détricotage du Brexit à la tentative de « reconstituer un œuf à partir d'une omelette » : $c^{\prime}$ est à Michel Barnier qu'il allait incomber de remplir cette mission quasiment impossible de refabriquer les œufs, avec des homologues généralement imprévisibles et parfois hostiles. Négociateur en chef de l'Union européenne, il s'était vu assigner la lourde tâche de gérer la sortie de la Grande-Bretagne de I'Union avec les représentants britanniques.

Les négociations furent délicates et semées d'embûches, et d'une complexité qui, souvent, dépassait la partie adverse. Celles-ci s'inscrivaient dans un contexte de relations anglo-européennes particulièrement difficiles, voire acrimonieuses, après quatre longues années d'un divorce qui n'en finissait pas de se concrétiser (Trouille 2020). Les négociateurs européens se retrouvaient face à un partenaire aux exigences aussi déraisonnables qu'irrationnelles, difficiles à accommoder sans risquer de compromettre les fondations du Marché intérieur, dictées par une idéologie nationalsouverainiste digne du XIX siècle, et dont la priorité semblait consister davantage à décrédibiliser l'Europe que de conclure un accord commercial sérieux. D'ailleurs, I'un des buts du Brexit n'était-il pas d'affaiblir l'Europe et sa structure de gouvernance, de contester ses valeurs, de tenter de diviser pour mieux régner ? Du point de vue de ses

* Professeur Jean Monnet en intégration économique européenne à l'École de Management de l'Université de Bradford au Royaume-Uni. Ses intérêts de recherche portent sur les relations économiques franco-allemandes, les politiques européennes, et plus récemment, sur les questions de développement et $d$ 'intégration régionale en Afrique dans une perspective comparative. Il dirige depuis 2016 le Réseau Jean Monnet « The European Union, Africa and China in the Global Age » (EU-EAC). j.m.l.trouille@bradford.ac.uk

* * Professeur associée à York St John University au Royaume-Uni, juriste et interprète. Elle détient un doctorat en procédures pénales comparatives. Ses publications portent sur les rapports entre la justice et les affaires et les réformes du système judiciaire. Ses recherches actuelles concernent le droit pénal international, l'accès à la justice et la réconciliation dans les États sortant d'un conflit. h.trouille@yorksj.ac.uk 
protagonistes les plus fervents, le Brexit ne pouvait être un succès que s'il s'accompagnait d'un échec du projet européen. Telle une épée de Damoclès, cette toile de fond pouvait à tout moment assombrir la scène, semer la zizanie parmi les État-membres, briser le consensus sur des acquis communautaires essentiels, et remettre ainsi en cause les fondements mêmes du projet d'intégration européenne.

\section{La grande illusion}

La tâche de Michel Barnier allait donc s'avérer rude, et tout particulièrement au cours des six derniers mois de la période de transition accordée aux Britanniques. Jamais les enjeux d'une négociation commerciale n'avaient présenté de tels risques pour la cohésion de l'Union. De plus, jamais de telles négociations n'avaient eu pour but de remettre en question des accords commerciaux acquis de longue date et dont l'impact sur les économies des États-membres, Royaume-Uni inclus, s'était incontestablement révélé positif. La recherche d'un accord " gagnant-gagnant», but de toute négociation, devenait de fait celle d'un accord " perdant-perdant »: chacune des parties, sur la base d'argumentaires fondamentalement opposés, visait à atténuer les multiples effets collatéraux que les nombreuses « lignes rouges » imposées par le camp britannique rendaient inévitables. Ces lignes rouges avaient été tracées dès janvier 2017 par Theresa May dans son allocution de Lancaster House, à la stupeur de Michel Barnier qui l'avait écoutée dévoiler toutes ses cartes. Vu l'ampleur de ces lignes rouges, qu'allait-il rester à négocier ? Un accord de type norvégien, ou suisse, était perdu d'avance. Il en allait de même d'un accord sur le modèle de celui qui lie I'UE à l'Ukraine, ou à la Turquie... Dans ce jeu de dupes, le gagnant ne pouvait être que celui des deux protagonistes qui y perdrait le moins. Pleinement conscient des enjeux comme des écueils, Barnier, entouré d'une équipe d'une trentaine de juristes, d'économistes et d'experts bien rodés, allait devoir naviguer à vue en gardant le cap, c'est-à-dire définir des règles du jeu équitables, notamment en matière d'aides d'État, de réglementations du travail, de protection de l'environnement et d'impôts sur les sociétés, sans tomber dans les pièges que tendait la partie adverse, tout en s'assurant du soutien des Européens autour de leur principal bien commun, celui sur lequel Jacques Delors déclarait « on ne peut pas tomber amoureux d'un grand marché » : Michel Barnier, à force de ténacité et de pédagogie, est parvenu à préserver l'unité des 27 pays membres et de ressouder les Européens autour de leur Marché intérieur, ce marché que, comble d'ironie, le Royaume-Uni avait tant contribué à façonner.

Michel Barnier se réfère avec pertinence au célèbre film de Jean Renoir, La grande illusion, dans le titre de son Journal secret du Brexit qu'il vient de publier. La grande illusion est bien sûr celle du Brexit : I'illusion de la souveraineté, la poudre aux yeux de la grandeur retrouvée par une Global Britain qui se serait libérée de ses chaînes. Quant au film de Renoir, il prône la solidarité au-delà des divisions nationales ou de classe. Comment ne pas y voir un double message adressé aux Brexiters, qui rejettent la solidarité européenne pour entraîner leur pays dans une aventure insensée, dans un contexte international qu'Enrico Letta décrit comme " un monde de brutes » (Letta 2017). 


\section{Retour sur une négociation de tous les dangers}

Tandis que l'Allemagne, en juillet 2020, prenait les rênes de la présidence tournante du Conseil de l'Union européenne, la recherche d'un accord commercial UE-Royaume-Uni entamait sa dernière ligne droite. Le manque de progrès dans les discussions était notoire. Les cycles de négociations qui se suivaient depuis de longs mois ressemblaient à un dialogue de sourds. Rien n'avançait sur les questions de concurrence loyale dans les domaines sociaux, environnementaux, fiscaux, d'aides d'État, ou de la pêche. Il était pourtant vital pour l'UE d'assurer des règles du jeu équitables afin que Londres ne puisse pas instaurer un système de dumping à ses portes. Mais pour Bruxelles, ce blocage était révélateur des intentions de Londres : au vu de l'intransigeance et, souvent, de l'impréparation de la partie adverse, un accord de dernière minute semblait de plus en plus probable.

Le 9 septembre 2020, Boris Johnson lançait un projet de loi sur le Marché intérieur britannique qui remettait en cause le protocole nord-irlandais inclus dans l'accord de retrait du Royaume-Uni conclu fin 2019 avec I'UE. Le Premier Ministre britannique semblait alors prêt à passer outre ses propres engagements internationaux, pour minimiser les contrôles douaniers avec l'Irlande du Nord sans devoir renoncer au type de politique industrielle qu'il souhaitait mettre en place, interventionniste et basée sur les aides d'État, quitte à devoir renoncer à conclure un traité de libre-échange avec I'UE. Pour la Commission européenne, ce projet de loi constituait une violation du droit international. Ursula von der Leyen, Présidente de la Commission, lança une procédure d'infraction, parvenant toutefois, lors d'une visioconférence à relancer les discussions en vue $d^{\prime}$ un accord commercial. Cependant, bien vite les négociations achoppèrent à nouveau sur les mêmes sujets qui fâchent. Le 16 octobre, Boris Johnson insatisfait du refus de Bruxelles de concéder un accord de libre-échange sur le modèle de celui offert au Canada, déclarait qu'il " est désormais temps de se préparer à un no deal ». Certes, un "Canada Deal » offert à un pays tiers situé aux portes de l'Europe présentait un risque que I'UE n'était pas prête à courir sans s'assurer de solides garde-fous. Après une nouvelle période de blocage, les tractations reprirent toutefois, mais la possibilité d'aboutir à un accord semblait de plus en plus improbable. Toute tentative de compromis butait sur les lignes rouges tracées par le camp britannique : souveraineté, reprise de contrôle, négocier sur un pied d'égalité, étaient devenus les maîtres-mots, qu'il soit question de pêche dans les eaux territoriales du Royaume-Uni, de concurrence ou de gouvernance.

Dans un même temps, le 10 Downing Street suivait avec intérêt l'évolution des élections présidentielles américaines. Une réélection de Donald Trump aurait ouvert la voie à un accord commercial avec les États-Unis qui, même désavantageux, rendait un accord avec les Européens politiquement moins nécessaire aux yeux de Boris Johnson. Cependant, les incertitudes liées à cette hypothétique réélection du candidat Trump encouragèrent le gouvernement britannique à laisser une porte entrouverte aux Européens. Ces derniers, profondément agacés par le manque d'avancée des pourparlers, signifièrent à Londres qu'ils n'étaient plus en mesure de considérer $d^{\prime}$ autres concessions, et que la balle était désormais dans leur camp. Londres reprocha à l'UE, et notamment à la France, d'avoir ajouté de nouvelles demandes, ce qui fut démenti. Début décembre, alors que la date butoir du 31 décembre se rapprochait, les négociations restaient bloquées, tandis que les intentions de Londres 
restaient floues et que les deux parties semblaient s'acheminer vers un " no deal ». Bruxelles décida alors d'accélérer les préparatifs en cas de non-accord. Les discussions allaient néanmoins reprendre le 14 décembre dans un climat plus positif à la suite d'un entretien téléphonique qualifié de " constructif et utile » entre Ursula von der Leyen et Boris Johnson. Les deux parties se déclaraient soudain prêtes à négocier jusqu'à la dernière heure. C'est finalement à la veille de Noël que l'accord allait enfin être conclu, après plus de quatre années de négociations ardues visant tant bien que mal à mitiger les conséquences du divorce anglo-européen.

Pascal Lamy avait décrit ce détricotage comme "la première négociation de I'histoire où les deux camps partaient d'une base de libre-échange pour discuter de quelles barrières ils allaient ériger ». Cette improbable négociation, reflet de l'absurdité du Brexit, se matérialisait sous la forme d'un lourd document de 1200 pages qui, à l'heure d'écrire ces lignes quelques mois après sa mise en application, ne satisfait personne, et dont les conséquences se montrent désastreuses pour des pans entiers de l'économie britannique. Comment avait-on pu en arriver là ?

\section{Un choc des systèmes}

En son temps, Mario Monti, fréquemment confronté, comme Commissaire européen au Marché intérieur (1995-99) puis à la Concurrence (2000-2004), aux profondes divergences de vues qui opposaient Londres et le Continent, soulignait inlassablement la nécessité de réconcilier, au sein de l'Europe, deux visions antagonistes du rapport au marché. L'une continentale, avec ses variantes nationales, notamment allemande (ordolibérale) ou française (universaliste et corporatiste), mais toujours orientée sur l'économie sociale de marché, la valeur partenariale, et la recherche d'une répartition plus équitable de la production de richesses. Et l'autre britannique, reposant sur l'économie libre de marché, la finance et la valeur actionnariale (Hancké et al. 2007). Pour Monti, il était clair qu'il s'agissait d'une division idéologique. Seuls des compromis prudemment élaborés pouvaient rapprocher les deux grandes orientations économiques qui avaient dessiné les contours du Marché intérieur. Le rapport qu'il rédigea sur l'état du Marché intérieur (Monti 2010) invitait à des concessions de part et d'autre en vue de réduire les tensions qui opposaient ces deux principales variétés de capitalisme. Toutefois, la décision du Royaume-Uni en juin 2016 de quitter I'UE allait marquer l'échec de cette tentative louable, pourtant nécessaire pour l'intérêt européen, de les voir converger. Cette décision de s'extraire des réglementations européennes marquait aussi la consécration d'un antagonisme profond entre ces deux formes dominantes de capitalisme, engagées dans une lutte $d^{\prime}$ 'influence dont le Brexit marquait l'aboutissement.

En effet, le référendum de juin 2016 a amorcé un tournant majeur dans la rivalité qui oppose les deux principales formes de capitalisme. Depuis, le fossé qui sépare ces deux visions n'a cessé de se creuser. $D^{\prime}$ une part, le capitalisme continental, régulé, que certains qualifient de, domestiqué, s'accommode d'un État administrateur. II recherche la stabilité, la prévisibilité, et impose les règles et contrôles qui protègent les positions de marché face à de nombreux concurrents peu scrupuleux. D'autre part, la faction " anglo-saxonne », ou britannique, décrite par l'écrivain George Monbiot (2021) comme celle des chefs de guerre du capitalisme, a pour objectif de s'affranchir de toute contrainte régulatoire. Ces derniers, milliardaires américains, oligarques 
russes ou magnats des médias, tous généreux donateurs du Parti Tory, partagent une vision commune hostile à toute contrainte de marché. Ils considèrent l'imposition fiscale, que ce soit sur les sociétés ou sur les personnes, comme illégitime. Les mesures européennes de lutte contre l'évasion fiscale ne pouvaient que renforcer leur détermination d'affranchir le Royaume-Uni du cadre réglementaire de I'UE. Ils appréhendent la démocratie comme un obstacle incompatible avec la liberté des marchés. Dans la lutte d'influence entre États et marchés, ce dernier est perçu comme valeur absolve, qui ne peut résulter que d'un processus libéré de toute médiation ou contrainte. C'est le message que prône Steve Bannon, ancien stratège de Donald Trump, à qui veut l'entendre, de part et d'autre de l'Atlantique, de Marine Le Pen à l'Alternative für Deutschland, de Salvini aux caciques de I'ERG', dans sa croisade pour la déconstruction de l'État administrateur. C'est aussi celui prôné par le milliardaire américain gestionnaire de fonds spéculatifs Robert Mercer, généreux donateur du Parti Tory, qui avait largement subventionné le vote Leave dans la campagne référendaire, et principal actionnaire de l'ancienne société d'analyse de données Cambridge Analytica, lourdement impliquée dans le vote Leave, et dont il est avéré que les algorithmes ont visé des millions de profils Facebook en vue d'influencer leur choix de vote peu avant le référendum.

Dans ce bras de fer entre les deux systèmes, le Brexit marque à la fois une victoire de la vision anti-régulatoire du capitalisme et la radicalisation de cette même vision. Pour les tenants d'un capitalisme financier bénéficiant de toute latitude sur des marchés non soumis à des contraintes réglementaires, le Brexit représente une victoire inespérée, et même une incroyable aubaine. Le Royaume-Uni, pays doté d'une force financière et $d$ 'une influence internationale considérables (bien que fortement érodées dans la foulée du Brexit), et d'un siège permanent au Conseil de Sécurité des Nations Unies, devient ainsi leur tête de pont parmi le club restreint des nations les plus puissantes. L'image du Brexit véhiculée par les médias était celle d'une réaction face à un afflux d'étrangers, de réfugiés (pourtant très limité), un désir de "reprendre le contrôle » de ses lois et de rétablir le pays comme "nation indépendante et souveraine ». Tout l'éventail de l'argumentaire populiste fut déployé à cette fin sans réserve aucune, en vue de frayer la voie à un Brexit dur, seul susceptible d'affranchir le Royaume-Uni d'un maximum de règles européennes. Rendue possible par une manipulation à grande échelle avec la complicité d'une large part des médias, cette quête continue de produire un écran de fumée populiste dont le trop célèbre Nigel Farage, fréquemment sollicité par des radios et chaînes de télévision partisanes, et ses acolytes ne sont que les mercenaires d'un projet bien plus vaste. Sa finalité est d'imposer la prééminence d'un système capitalistique qui ne s'encombre ni de règles, ni de démocratie, au prix d'une refonte systématique de l'économie britannique, sans état d'âme à l'égard des innombrables victimes collatérales de cette expérience, des pertes d'emplois en masse, de tous ceux pénalisés par les difficultés d'accès au marché européen, sans oublier les jeunes, eux aussi grandes victimes de la fin des programmes Érasme et de la libre circulation, ni les retraités, pour lesquels s'installer dans un pays de l'UE devient beaucoup plus difficile et coûteux.

Le but ultime du Brexit, cheval de Troie de ce capitalisme débridé, est de renverser I'État administrateur et de remplacer ce dernier par une forme extrême de néolibéralisme

1. ERG : European Research Group, groupe militant ultra-conservateur et anti-européen au Parlement de Westminster. 
qui remette en question les droits sociaux, impose une politique de dumping fiscal, réglementaire et environnemental pour s'imposer comme une économie aussi dérégulée que possible et comme le paradis fiscal aux portes de l'Europe. Lorsque, en réponse aux inquiétudes formulées par des entrepreneurs sur l'impact économique qui s'ensuivrait d'un Brexit dur, Boris Johnson répondait, excédé, en juin 2018 : Fuck business! (littéralement : " que les entreprises aillent se faire foutre! »), oubliant la réputation de parti des entreprises que les Tories avaient acquise depuis Margaret Thatcher, Johnson pensait aux entreprises qui comptent sur les protections de l'État administrateur. Sa remarque pour le moins crue ne concernait pas les corporations et intérêts de ses alliés politiques. Ce n'est pas le principe schumpeterien de destruction créatrice naturelle qui allait sélectionner les meilleures entreprises dans la foulée du Brexit, mais bien des choix politiques basés sur les relations des entreprises avec le pouvoir central, la générosité de leurs donations au parti conservateur, ou d'autres formes de soutien pour les maintenir au pouvoir. II n'est d'ailleurs pas rare qu'en préalable à toute concertation sur la possibilité d'octroyer une aide d'État, des entreprises se voient demander directement si elles soutiennent le Brexit. Tel est le rôle assigné à Boris Johnson, à son cabinet, à tout l'appareil du n ${ }^{\circ} 10$ Downing Street, à des think tanks et d'autres groupes d'intérêt. Tel est aussi le but des barons de la presse, souvent milliardaires, de Lord Rothermere (propriétaire du Daily Mail, de Metro et de " $i$ ») à Rupert Murdoch (qui détient le Sun et le Times), sans oublier Richard Desmond (Daily Express, Star), les frères Barclay (Daily Telegraph) et Lord Lebedev, oligarque russe et généreux contributeur du parti conservateur, récemment nommé à la Chambre des Lords lqui détient le Standard) : la tâche commune de ces nombreux acteurs est de préparer le terrain en vue d'une refondation systémique, et cette nouvelle infrastructure est mise en place par des ramifications qui s'étendent bien au-delà du gouvernement britannique.

\section{Brexit Britain et corruption : Le bateau ivre}

Cinq mois après son saut dans l'inconnu, le pays est plus divisé que jamais. L'Écosse, qui se sent trompée par le Brexit, envisage un retour au sein du giron européen comme nation indépendante. Les conflits du passé resurgissent en Irlande du Nord, où un rattachement à la République d'Irlande devient une option crédible. Même le Pays de Galles, la troisième nation celte d'un Royaume de plus en plus désuni prend ses distances de l'Angleterre quand elle le peut. Un profond malaise a envahi la société britannique (Bermann 2021), relayé par une cascade incessante d'affaires de corruption, un manque de transparence, et des types de comportement de personnalités politiques en vue qui, en temps normal, auraient fait l'objet d'appels à démission, mais sont soit minimisés par les médias qui n'y voient que des tempêtes dans des tasses de thé, soit balayées sous le tapis. Accusations de harcèlement sexuel et de viol perpétré par un membre conservateur du Parlement sur une stagiaire et enquête policière abandonnée malgré un bon nombre de témoins non interrogés, fréquentes intimidations de hauts fonctionnaires au Home Office (Ministère de I'Intérieur), influence répréhensible de donateurs au parti conservateur, manipulations politiques de procédures judiciaires, contrats juteux délivrés sans la moindre concurrence ni supervision à des parents ou amis de membres du gouvernement ou donateurs du parti Tory, sont devenues monnaie courante d'un pays dont le niveau de corruption s'apparente désormais davantage à celui d'États voyous qu'à celui d'une nation moderne et responsable mère de la Magna Carta. Certes, le classement annuel 2020 
de Transparence internationale (2020) situe le Royaume-Uni en onzième position sur I'indice de perception de la corruption, score comparable à l'Allemagne (9/180) et meilleur que celui de la France (23/180). Cependant, cet article s'intéresse à l'augmentation quasiment exponentielle de la corruption dans les hautes fonctions, où elle est en passe de devenir endémique, et non dans le secteur public (santé, éducation, justice...) où la corruption reste exceptionnelle. Mais on peur s'attendre à ce que le score crédité au Royaume-Uni reflète ce nouveau contexte dans un proche avenir.

Les cas les plus frappants d'irrégularités concernent l'attribution par des ministres du gouvernement à des relations et amis proches de contrats pour la fabrication d'équipements de protection individuels (EPI) pendant la pandémie de coronavirus. Les contrats attribués sont si nombreux qu'il est difficile de tous les nommer. Ils font l'objet de plusieurs poursuites judiciaires essentiellement menées par une organisation de juristes bénévoles, the Good Law Project, et l'association de médecins Every Doctor, en réaction au refus de représentants du gouvernement de justifier les raisons pour lesquelles certaines compagnies privées se sont vu attribuer des contrats lucratifs préférentiels au détriment $d^{\prime}$ autres firmes pourtant plus à même de fournir le matériel. Ces compagnies, souvent sans expérience en matière $d^{\prime} E P I$, parfois en situation de faillite, parfois même fictives ou nouvellement créées, ont pour point commun d'être possédées par des proches des ministres concernés et par de généreux contributeurs aux caisses du parti Tory ${ }^{2}$. En mars 2021 la Cour jugea que le gouvernement avait enfreint la loi en manquant à son obligation de rendre publics les contrats liés au coronavirus $^{3}$. Fait sans précédent, le Open Government Partnership ${ }^{4}$, une organisation globale pour la transparence et la lutte anti-corruption, a placé le Royaume-Uni sous observation pour ses pratiques en matière de transparence ${ }^{5}$ et, entre autres, pour avoir manqué de rendre publics les détails de contrats attribués dans les délais stipulés (Freedom of Information, 2021 : 17).

L'état de délabrement des valeurs morales dans la gestion du pays (Oborne 2021) est tout aussi évident dans l'attribution de contrats apparemment simples d'approvisionnement en repas gratuits pour écoliers de familles défavorisées en période de vacances scolaires. Ces contrats furent distribués sans appels d'offres concurrentiels, à des compagnies ayant elles aussi des liens avec des membres du parti conservateur, et qui furent sévèrement critiquées pour la qualité plus que médiocre des repas fournis (Campbell et Weale 2021). II en alla de même pour des contrats d'approvisionnement d'ordinateurs portables destinés aux écoles afin de permettre l'enseignement à distance (Carr 2020).

Alors qu'émergeaient ces scandales, un autre couvait dans l'ombre, lorsque Carrie Symonds, financée de Boris Johnson, revint à Downing Street après la naissance de leur enfant, et entreprit de redécorer l'appartement du Premier Ministre au numéro 11.

2. C'est notamment le cas de Pestfix, qui reçut pour environ 350 millions de livres de contrats, mais que le gouvernement présentait comme une compagnie "spécialisée dans les produits de lutte anti-parasitaire ». C'est encore le cas de Clandeboye Agencies, fabricant, selon les registres des compagnies, de « produits de confiserie et de chocolaterie », qui reçut pour 108 millions de livres de contrats de fabrication d'EPI. Voir Good Law Project (2021) EXCLUSIVE : 4 more VIP-lane companies revealed. Accessible sur : https://goodlawproject. org/update/awarded-contracts-vip-lane/ (Consulté le 6 mai 2021).

3. R (Good Law Project and ors) v the SOS for Health and Social Care [2021] EWHC 346 (Admin).

4. Partenariat pour un gouvernement ouvert.

5. Selon le Open Government Partnership, le Royaume-Uni n'a pas respecté son engagement de renforcer transparence et responsabilité. Le Royaume-Uni est le $9^{e}$ membre de ce groupe international à être placé sous observation après des pays comme la Bulgarie, le Malawi, Malte et l'Afrique du Sud. 
L'allocation annuelle de 30000 livres n'était apparemment pas suffisante pour des rénovations qui s'élevaient à 200000 livres, et une source de financement supplémentaire s'avérait nécessaire pour couvrir les frais. Depuis sa prise de fonction comme Premier Ministre, Johnson s'était souvent plaint de la difficulté de « joindre les deux bouts " sur un salaire deux fois moindre que celui qu'il recevait du Daily Telegraph comme éditorialiste. En avril 2021, des allégations émergèrent selon lesquelles une somme substantielle avait été avancée par le parti conservateur pour couvrir les frais de rénovation, argent que Johnson aurait reçu de Lord Brownlow sans que Johnson ne suivre la procédure l'obligeant à déclarer dans les 28 jours tout prêt ou donation susceptible d'influencer ses décisions. Malgré l'insistance de Johnson à souligner qu'il avait payé la note, la Commission Électorale lança une enquête, considérant qu'il y avait de bonnes raisons de soupçonner qu'il y avait eu malversation. Le couple JohnsonSymonds fait déjà l'objet $d$ 'une enquête pour avoir bénéficié de vacances financées par des donateurs sur l'île Moustique, dans les Antilles (Durrant et al. 2021), et il a été révélé récemment que le couple a demandé une aide financière aux donateurs du parti conservateur pour financer une nourrice pour leur bébé (Wood 2021).

Juste avant que n'éclate ce scandale de la rénovation de l'appartement ministériel, d'autres révélations, plus sérieuses encore, se firent jour, dont la gravité laissa même penser à certains que la divulgation de l'affaire du flatgate ne serait qu'une diversion pour la dissimuler. En avril, I'ancien premier ministre conservateur David Cameron était exposé pour démarchage auprès du gouvernement au profit de Greensil Capital, compagnie dans laquelle Cameron détenait des millions de livres d'actions, dirigée par son ancien conseiller devenu son employeur. Le ministre de la Santé Matt Hancock était également impliqué, et il fut révélé qu'il avait reçu des actions dans une compagnie en février 2019, et que cette firme avait obtenu le mois suivant un contrat avec le National Health Service (NHS) (Walker 2021). Peu de temps plus tard, dans un échange de SMS entre Johnson et le milliardaire britannique Dyson communiqué aux médias, Johnson s'engageait personnellement à « régler » une question d'impôts qui touchait les affaires de Dyson.

Nombreuses sont les affaires de copinage, de népotisme et de corruption qui se révèlent presque quotidiennement. L'une d'elles, particulièrement choquante, concerne la nomination de Dido Harding, amie de David Cameron et épouse du député conservateur John Penrose, pour prendre en charge la mise en place d'un service Test and Trace ${ }^{7}$, lancé en mai 2020. Quelque temps plus tard, Test and Trace dut admettre que leur service n'employait pas moins de 2500 consultants, rémunérés chacun à un taux journalier moyen de 1100 livres, et que le salaire journalier le plus élevé atteignait 6624 livres. Malgré ces dépenses considérables et des salaires exorbitants, Test and Trace fut déployé des mois de retard par rapport à de nombreux pays qui bénéficiaient déjà $d$ 'un système équivalent, et ses performances ont été pour le moins médiocres pour un projet auquel avait été allovée la somme incroyable de 37 milliards de livres sterling d'argent public sur deux ans. Le Commons Public Account Committee ${ }^{8}$ avait émis des doutes sur l'efficacité du système, ajoutant qu'il n'était pas démontré que sa contribution à la réduction des niveaux d'infection ait justifié son coût (Parliament. House of Commons, 2019-21). Le fait que Harding fait partie du Comité exécutif

\footnotetext{
6. Service National de la Santé.

7. Test et traçage anticovid.

8. Comité de surveillance des comptes publics au parlement, composé de représentants de tous les partis.
} 
du Jockey Club, propriétaire de Cheltenham Racecourse, n'est pas non plus passé inaperçu. Les courses de Cheltenham, début mars 2020, avaient attiré des foules de 60000 personnes par jour sur quatre jours, au moment où la pandémie commençait à se faire sentir au Royaume-Uni, alors que de nombreux appels avaient été lancés pour que cet évènement soit annulé. À la suite de ces courses, les professionnels de santé ont identifié un foyer de coronavirus dans cette région, et le professeur Tim Spector, qui dirige le projet de traçage le plus large du pays, déclara que ces quatre journées et les contacts qui s'ensuivirent avaient causé « une augmentation de décès et de souffrances » en raison de la décision de laisser ces courses avoir lieu (France 24, 2020). Par un surprenant fait du hasard, le Haut Responsable de lutte contre la corruption du gouvernement britannique, John Penrose, n'est autre que le mari de Dido Harding.

L'affaire Harding n'est qu'un exemple parmi une multitude d'autres cas qui montrent à quel point la gestion de la pandémie par le gouvernement Johnson s'est révélée catastrophique. D'abord prise avec désinvolture et cynisme, et non sans considérer un temps la possibilité de laisser se créer une immunité collective dont les conséquences humaines auraient été catastrophiques, I'infection de Covid-19 se présentait comme une aubaine qui allait permettre au gouvernement de dissimuler l'impact économique et social du Brexit, lequel ne manquerait pas de frapper le pays après la fin de la période de transition. Mais avec 128000 morts officiels début mai 2021 (mais dans les faits probablement davantage), the Royaume-Uni compte l'un des taux de mortalité liée au coronavirus les plus élevés au monde en proportion de sa population. Un autre cas est aussi révélateur d'une profonde hypocrisie : celui de Dominic Cummings, ancien conseiller spécial de Boris Johnson, accusé de ne pas avoir respecté les règles de confinement en mars 2020 dans un déplacement de $432 \mathrm{~km}$ de Londres à Durham, avec sa femme et leur fils, tous deux atteints du Covid. Cummings argumenta, dans une discussion télévisée transmise du jardin de roses de Downing Street, que ce voyage avait été nécessaire pour organiser la garde de leur enfant au cas où lui et sa femme tombent malades. De nombreux témoins les virent séjourner dans une maison familiale près de Durham et visiter la région. Tandis que de nombreuses personnes ont eu des amendes pour motifs moins graves, Cummings bénéficia du plein support de Johnson. Toutes les tentatives à ce jour de le poursuivre pour infraction ont échoué (Weaver 2020). Des investigations sur sa propriété de Durham ont mis en évidence que Cummings n'a pas payé d'impôts locaux, mais n'a pas été inquiété.

\section{Conclusion}

La fin de la Présidence allemande de l'Union européenne, au 31 décembre 2020, marquait aussi celle de la période de transition, que Boris Johnson avait systématiquement refusé de prolonger malgré les conditions posées par la pandémie. Elle marquait la sortie définitive du Royaume-Uni du Marché intérieur et de l'union douanière. Au cours de ces six mois, l'Allemagne a joué un rôle de soutien à Michel Barnier, notamment lorsqu'il fallait s'assurer de la solidarité de tous les États membres de I'Union. C'est tout l'art et la détermination de Barnier que d'avoir réussi, à grand renfort de patience, de ténacité et de pédagogie, à contenir ces forces hostiles au projet européen pendant la durée des négociations et d'avoir abouti à un accord. Cet accord est certes limité, et ne dispense pas les compagnies qui commercent avec les deux rives de la Manche de se plier à de nouvelles formalités douanières, sanitaires, 
phytosanitaires, à des déclarations d'importation ou d'exportation, d'autorisations de transit pour le conducteur, de certificats de conformité et de TVA. Mais il s'agit probablement du meilleur accord qui puisse être atteint dans les paramètres tracés par les lignes rouges de chaque camp.

Le Royaume-Uni et l'UE se retrouvent donc avec un accord commercial sans aucune mesure avec les avantages considérables qu'offrait l'appartenance à l'Union. Mais au vu des circonstances, Michel Barnier le présente comme un accord utile et équilibré autour de quatre piliers. Un accord de libre-échange équitable. Un partenariat économique qui couvre les transports, l'énergie, la lutte contre les changements climatiques et la pêche, une coopération en matière de recherche et $d^{\prime}$ 'innovation, de sûreté nucléaire et pour l'espace dans le cadre des programmes. Un troisième pilier concerne la sécurité des citoyens et la lutte antiterroriste. Quant au dernier pilier, il concerne la gouvernance de cet accord (Barnier 2021). Car les dirigeants européens, allemands et français ont dû, et devront continuer à être vigilants face à la possibilité que le Royaume-Uni cherche à nuire à I'UE. Sa capacité de nuisance est certes relativement limitée. Mais cela ne signifie pas qu'il ne soit pas en mesure de causer des dommages sérieux. L'envoi de deux frégates de la Royal Navy à Jersey pour un différend prévisible sur les droits de pêche, quelques jours seulement après la ratification par le Parlement européen de l'accord, et trois jours avant la Journée de l'Europe, $\mathrm{n}^{\prime}$ augure rien de bon, même si la finalité de sortir la canonnière était probablement de faire le plein des voix populistes lors des élections locales, le même jour au RoyaumeUni. Lucide, Barnier lance un avertissement aux Européens : les provocations sur le protocole irlandais ne vont pas s'arrêter. Le gouvernement britannique tentera d'effacer les conséquences du Brexit, dont il est responsable. La tentation sera forte de se réintroduire dans le Marché intérieur, dont il a pourtant claqué la porte. Il faudra rester vigilent face à toute forme nouvelle de cherry-picking'. De même, la tentation sera forte de mettre à profit ses nouvelles marges de manœuvre pour s'octroyer, secteur par secteur, des avantages compétitifs. À chaque tentative se posera la question de savoir si ces avantages reposent sur une concurrence loyale et ouverte, ou sur une politique non coopérative qui mène à un dumping social, économique ou fiscal aux dépens de l'intérêt européen. Mais l'avertissement final de Barnier porte sur l'Europe elle-même. Rester vigilant, c'est aussi se garder de toute certitude ou arrogance, avoir conscience de nos propres faiblesses, savoir écouter les sentiments populaires qui s'expriment un peu partout en Europe, et savoir leur apporter une réponse (Barnier 2021).

Il est frappant de constater, à chaque ligne de La grande illusion, qu'à aucun moment Barnier ne se laisse aller à de basses critiques du Royaume-Uni, pays dont il admire profondément l'histoire, la culture, et ses innombrables apports à l'Europe. Dans le climat délétère et acrimonieux créé par le Brexit et, souvent, la mauvaise foi du camp adverse, il aurait pu en être tout autrement. C'est la marque d'une grande personnalité politique à la hauteur des circonstances, qui a eu la sagesse de ne pas se laisser dominer et a su rester maître d'un rapport de force difficile.

Dans cette nouvelle configuration de l'Europe, et face à la tectonique des plaques géopolitiques planétaires, Global Britain se retrouve isolée. Sur le plan intérieur, le Royaume-Uni est un pays plus divisé que jamais, où ce n'est ni plus ni moins que la démocratie qui est en jeu. Le Brexit, coup politique réalisé à grand renfort de

9. Appropriation. 
manipulations de tous ordres, mais conçu pour ne servir que les intérêts d'une petite minorité privilégiée au détriment de la grande majorité, a induit une réduction massive et systématique des droits des citoyens et des protections juridiques. La démocratie britannique est dysfonctionnelle et permet au parti conservateur, reconfiguré par Boris Johnson en parti populiste autoritaire, nationaliste, et largement soutenu par les médias, de se faire élire sans majorité grâce au système uninominal majoritaire à un tour, un découpage électoral qui lui est fortement avantageux et que seule une coalition des principaux partis d'opposition permettrait de battre.

On peut espérer que, dans la tourmente post-Brexit, le Royaume-Uni, tel le bateau ivre d'Arthur Rimbaud gagné par l'ivresse de la liberté, mais qu'une lame de fond semble entraîner vers le déclin, ne se ressaisisse enfin, et que la violence du naufrage, comme dans le poème, le mène vers la délivrance. Peut-être songera-t-il alors, nostalgique, au vers 84 : Je regrette l'Europe aux anciens parapets !

\section{Références}

Barnier, M. (2021) La grande illusion. Journal secret du Brexit (2016-2020), Gallimard, Paris.

Bermann, S. (2021) Goodbye Britannia : Le Royaume-Uni au défi du Brexit, Les essais, Stock, Paris.

Campbell, L. et Weale S. (2021) «Rashford: something "going wrong" with free school meal deliveries », The Guardian, 12 janvier.

Carr, J. (2020) «£96m laptop contracts went to Tory donor's firm», Schools Week, 9 octobre.

Durrant, T., Pannell, J. et Haddon, C. (2021) « Investigations into the Downing Street flat refurbishment », Institute for Government. 30 Avril. Accessible à https://www. instituteforgovernment.org.uk/explainers/investigations-downing-street-flat-refur bishment (accédé le 2 mai 2021).

France 24 (2020) Scientist slams decision to hold Liverpool match and Cheltenham Festival (26 mai) accessible à https://www.france24.com/en/20200526-scientist-slamsdecision-to-hold-liverpool-match-and-cheltenham-festival (accédé le 4 mai 2021).

Freedom of Information (2021) «Open Government Partnership puts UK under review » 17(4), 17-18.

Good Law Project (2021) EXCLUSIVE : 4 more VIP-lane companies revealed. Accessible sur : https://goodlawproject.org/update/awarded-contracts-vip-lane/ (accédé le 4 mai 2021).

Hancké, B., Rhodes, M., Thatcher, M. (dir.) (2007) Beyond Varieties of Capitalism : Conflict, Contradictions and Complementarities in the European Economy, Oxford University Press, Oxford.

Letta, E. (2017) Faire l'Europe dans un monde de brutes, Fayard, Paris.

Monbiot, G. (2021) «Brexit stems from a civil war in capitalism - We are just collateral damage », The Guardian, 24 novembre.

Monti, M. (2010) Une nouvelle stratégie pour le Marché unique. Au service de l'économie et de la société européennes. Rapport au Président de la Commission européenne José Manuel Barroso, présenté le 9 mai 2010. https://www.europarl.europa.eu/ 
document/activities/cont/201005/20100517ATT74639/20100517ATT74639FR. pdf (accédé le 2 mai 2021).

Oborne, P. (2021) The Assault on Truth : Boris Johnson, Donald Trump and the Emergence of a New Moral Barbarism, Simon \& Schuster, London.

Parliament. House of Commons Public Accounts Committee (2019-21) COVID-19 : Test, track and trace (part 1) (HC 932).

Transparency international (2020). Accessible sur : https://www.transparency.org/en/ countries/united-kingdom (accédé le 16 mai 2021).

Trouille, J-M. (2018) "L'impact du Brexit sur la relation franco-allemande», Allemagne $d^{\prime} a u j o u r d ' h u i$, octobre-décembre, $n^{\circ} 226$, Lille, 74-84.

Walker, P. (2021) «Greensill inquiry : David Cameron and founder to give evidence to MPs'», The Guardian, 7 mai. Accessible sur : https://www.theguardian.com/business/ 2021 /may/07/greensill-inquiry-david-cameron-and-founder-to-give-evidence-to-mps (accédé le 3 mai 2021).

Weaver, M. (2020) «People won't forget Dominic Cummings' visi' : Barnard Castle learns to live with notoriety », The Guardian, 17 December. Accessible sur : https://www. theguardian.com/lifeandstyle/2020/dec/17/people-wont-forget-dominic-cummingsvisit-barnard-castle-learns-to-live-with-notoriety (accédé le 5 mai 2021).

Wood, V. (2021) « Tory donors approached to pay for Borins Johnson's nanny and personal trainer, reports say », The Independent. 2 May. Accessible sur: https://www. independent.co.uk/news/uk/politics/boris-johnson-nanny-flat-loan-b 1840805.html (accédé le 3 mai 2021). 